\title{
Epizoic amoebae from the gills of turbot Scophthalmus maximus
}

\author{
Iva Dyková1,*, Antonio Figueras ${ }^{2}$, Beatriz Novoa ${ }^{2}$ \\ ${ }^{1}$ Institute of Parasitology, Academy of Sciences of the Czech Republic, Branišovská 31, 37005 Ceské Budějovice, Czech Republic \\ ${ }^{2}$ Concejo Superior de Investigaciones Cientificas, Instituto de Investigaciones Marinas, Eduardo Cabello 6, 36208 Vigo, Spain
}

\begin{abstract}
Species of amoebae belonging to the genera Platyamoeba Page, 1969, Vannella Bovee, 1965 and Flabellula Schaeffer, 1926 were found to accompany Paramoeba sp., the agent of amoebic gill disease (AGD), in clinically diseased turbots. The same community of epizoic gymnamoebae was found on the gills of turbots which revealed no gill abnormalities but slight behavioral signs indicative of suboptimal health status. The assemblage of the above-mentioned free-living amoebae capable of colonizing gill tissue of turbots was supplemented with species recognized in samples fixed from primary isolates for transmission electron microscopy. The pathogenic potential of epizoic gill amoebae in turbots is discussed.
\end{abstract}

KEY WORDS: Epizoic amoebae - Gill infection Scophthalmus maximus - Mariculture

\section{INTRODUCTION}

Amoebic gill disease (AGD) of turbot Scophthalmus maximus (L.) was diagnosed for the first time in 1995 (Dyková et al. 1995). Due to difficult cultivation, generic assignment of the agent was published later (Dyková et al, 1998a), along with a note on other strains of free-living amoebae which accompanied Paramoeba sp. In the same year Leiro et al. (1998) presented a study of Platyamoeba sp., which they claimed caused AGD in farmed turbots from the same region as reported in previous papers of Dyková et al. (1995, 1998a). Unfortunately, histopathology of AGD was not studied in detail by Leiro et al. (1998), and the alleged agent of $A G D$ was shown in situ in a single semithin section of a small sample of gill tissue.

The aim of this communication is to disclose the diversity of amoeboid organisms capable of colonizing the gills of turbots.

\section{MATERIALS AND METHODS}

The data presented are based on 2 successive screenings in turbot sea farms in 1996 and 1998. The

•E-mail: iva@paru.cas.cz object of the first screening was the group of clinically diseased turbots as described in the paper presenting the agent of AGD (Dyková et al 1998a). The second screening covered a group of the same hosts with clinically healthy gills. In the first screening, 14 turbots from the stock with AGD were sampled; 4 of these had clinical symptoms of AGD. The second screening was carried out in 2 farms and included a total of 72 turbots: the gills of 24 turbots of the size category 12 to $20 \mathrm{~cm}$ were sampled in farm No. 1 , which experienced AGD in 1994 (Dyková et al. 1995), and 48 turbots were sampled in farm No. 2, where mortalities caused by AGD occurred in 1996. This group of turbots of the size category 12 to $15 \mathrm{~cm}$ comprised 20 specimens with optimal health status and 28 with slight behavioral signs indicative of suboptimal health condition. No gill abnormalities were detected in this group of fish. Amoebae isolated from samples of turbot gill tissue were characterized and compared. In total, 53 strains were examined with the light microscope, and 27 of them were also examined with the electron microscope. The methods of isolation, culture and cloning as well as fixation for transmission electron microscopy (TEM) were the same as described in previous papers (Dyková et al. 1995, 1998b). Some of the cultures in liquid medium as well as on agar plates were maintained for several weeks only, after which they per- 
ished due to bacterial overgrowth. Non nutrient agar (Bacto agar, Difco) used for subculturing was seeded with autoclaved Pseudomonas sp.

\section{RESULTS}

In addition to Paramoeba sp., the agent of AGD, 20 strains of other amoebae were isolated from the gill tissues of 7 out of 14 turbots sampled during the first screening in 1996 in a turbot farm in Northwest Spain. These 7 positive turbots included 4 with clinically manifested AGD.

Twenty amoeba strains were isolated from the gills of 17 out of 24 turbots examined in 1998 from farm No. 1. Thirteen strains, including 5 Paramoeba strains, were isolated from the gills of 8 out of 28 examined turbots, which in farm No. 2 revealed no gill changes and only slight behavioral alterations. Isolation attempts in the group of 20 turbots with certified optimal health status from farm No. 2 gave negative results.

Among 53 strains isolated from the gills of 32 out of 86 examined turbots, the most numerous (48 strains) were flattened (discoid or fan shaped) amoebae. They could easily be distinguished from Paramoeba by their shape and a well-developed hyaloplasmic region in trophozoites (Figs. 1 to 15). While on agar plates, trophozoites of individual strains revealed polymorphism, especially in the zones of intensive multiplication; the shape of trophozoites observed in hangingdrop preparations was more or less uniform. Shape divided strains of flattened amoebae into 2 groups. The first group included strains with trophozoites possessing a veil-like extensive hyaloplasmic region (Figs. 1 to 13) with a smooth rim. The trophozoites of the second group were characterized by an irregular outline of hyaloplasm (Figs. $14 \& 15$ ).

Taxonomic analysis based on light microscopy and TEM of 22 clonal strains representative of small, medium, large and giant size categories of flattened trophozoites revealed the presence of species belonging to 3 genera. Sixteen out of 22 analysed strains of flattened amoebae were assigned to the genus Platyamoeba Page, 1969. Ten out of 16 Platyamoeba strains were assigned to the group of small and medium sized species of the genus which includes, e.g. P. longae Sawyer, 1975, P. murchelanoi Sawyer, 1975, P. weinsteini Sawyer, 1975, P. douversi Sawyer, 1975, P. leei (Sawyer, 1975) Page, 1983 and P. plurinucleolus Page, 1974 listed in Page $(1980,1983)$. The greatest dimensions of trophozoites of these clonal strains (Figs. 1. to 7 ) did not exceed the range 11.4 to $21.5 \mu \mathrm{m}$. Trophozoite size allowed 6 out of 16 analysed Platyamoeba strains to be categorized in the group of large and giant species (Figs. 8 to 11 ) which have a range of greatest dimension between 22.0 and $45.0 \mu \mathrm{m}$ (such as, e.g. P. bursela Page, 1974; P. mainensis Page, 1971; P. flabellata Page, 1974; P. australis Page, 1983; and $P$. calycinucleolus Page, 1974). All the mentioned species were described from localities distant from the area of our studies. Similarly, Page (1983) stressed a great intraspecific variation in Platyamoeba at light and electron microscopical levels. This, along with our own experience, confines our assignment of the clonal strains to the generic level only until molecular analysis is done. This idea was supported by TEM investigation which revealed slight differences in the thickness of the glycocalyx of individual strains (Figs. 17 to 19). Culture conditions were identical and the differences in the thickness of the glycocalyces could be attributed to different methods of fixation rather than real differences in ultrastructure of individual clones.

Strains belonging to the genus Vannella Bovee, 1965 were less numerous: 4 out of 22 strains of flattened amoebae analysed with TEM were assigned to this genus. All of them accompanied the agent of AGD. Although the greatest dimensions of trophozoites and the type of glycocalyx (Figs. 12,13 \& 16) complied with the size range of $V$. caledonica Page, 1979, V. septentrionalis Page, 1980 and $V$. anglica Page, 1980, we also restricted the diagnosis to the generic level until nonmorphological criteria can be applied.

Two out of 22 analysed clonal strains were assigned to the genus Flabellula Schaeffer, 1926 (Figs. 14 \& 15). The greatest dimensions of the trophozoites fitted in the range given by Page (1983) for trophozoites of $F$. citata Schaeffer, 1926 or $F$ calkinsi (Hogue, 1914).

Five Paramoeba strains were isolated from the gills of 5 turbots with slight behavioral abnormalities. Three strains were successfully cloned and subcultured. When observed on the surface of agar plates and in hangingdrop preparations, trophozoites were rather flattened, with a narrow hyaline zone and digitiform pseudopods. One or 2 parasomes were observed in the close vicinity of the nucleus. The active locomotive forms were longer than broad, having a minimum length of 14 to $15 \mu \mathrm{m}$ and a maximum length of 27 to $33 \mu \mathrm{m}$ (Figs. 20 to 22 ). The average breadth was 6.0 to $8.7 \mu \mathrm{m}$. Floating forms had a rounded central mass and long radiate pseudopods. No cysts were formed by any of these strains. The basic diagnostic features as well as details of ultrastructure observed in 3 Paramoeba strains (Fig. 23) were identical with $P$. pemaquidensis Page, 1970. When compared with trophozoites of 2 strains of $P$. pemaquidensis isolated from the Mediterranean area and cultured under the same conditions, a difference in size of trophozoites but not in ultrastructure was noticed. The average length and breadth of the 3 Paramoeba strains under study were smaller than those of the Mediterranean strains, but larger than 

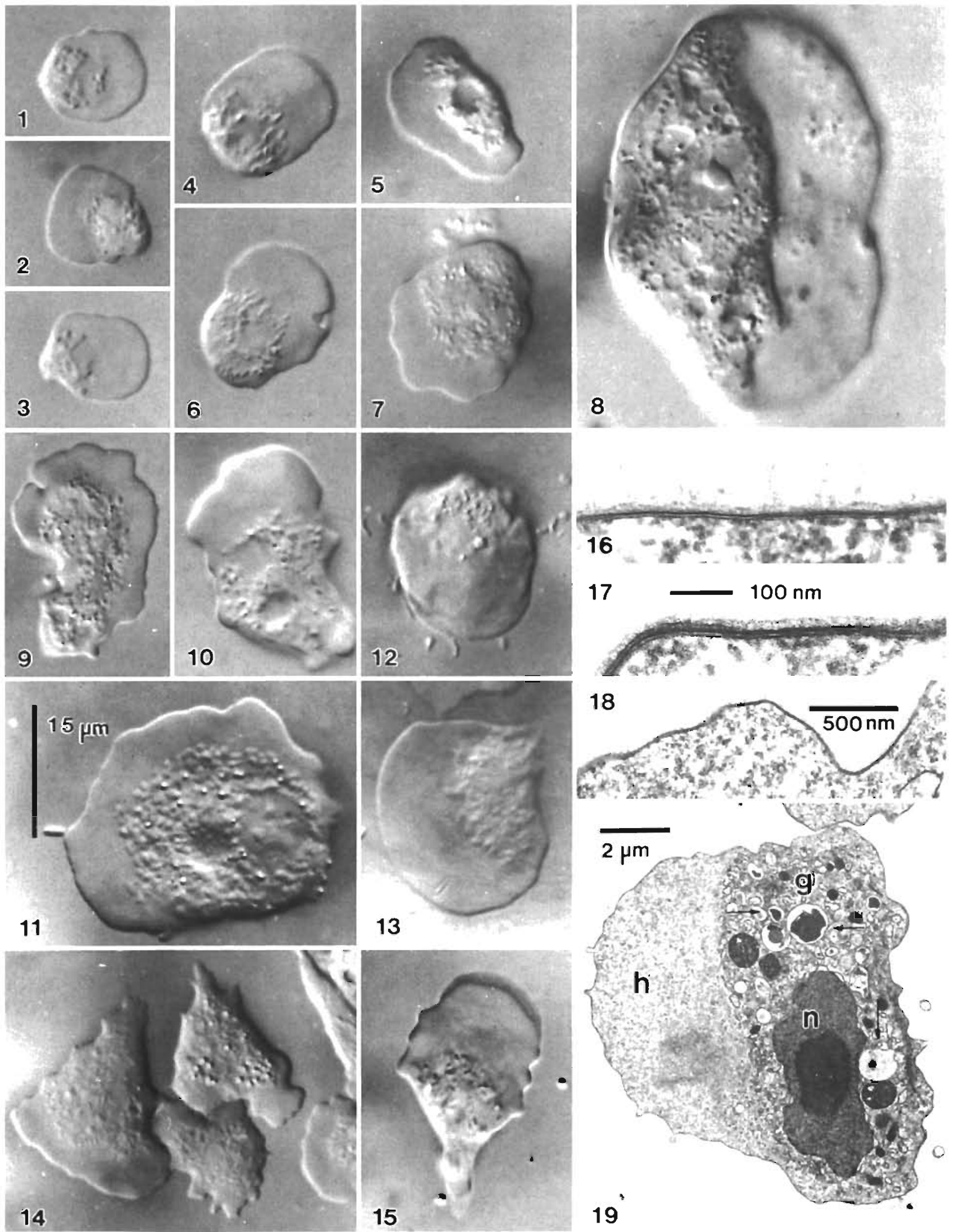

Figs. 1 to 19. Trophozoites of flattened amoebae isolated from gills of turbots. Figs. 1 to 7 . Trophozoites of Platyamoeba spp., representatives of the group of small and medium sized species of this genus, as seen in hanging-drop preparations. Figs. 8 to 11. Platyamoeba trophozoites belonging to the group of large and giant species. Figs. 12 \& 13 . Trophozoites of Vannella spp. isolated from gills of turbots together with the agent of amoebic gill disease (AGD). Figs. $14 \& 15$. Trophozoites of 2 Flabellula strains. Scale bar in Fig. 11 is valid for Figs. 1 to 15. Fig. 16. Glycocalyx of Vannella spp. with glycostyles extending up to $135 \mathrm{~nm}$ above the plasma membrane. Figs. 17 \& 18. Cell surface of 2 Platyamoeba strains. Fig. 19. Ultrathin section through Platyamoeba trophozoite showing granuloplasm $(\mathrm{g})$ with nucleus $(\mathrm{n})$, numerous vacuoles (arrows) and hyaloplasm $(\mathrm{h})$ 


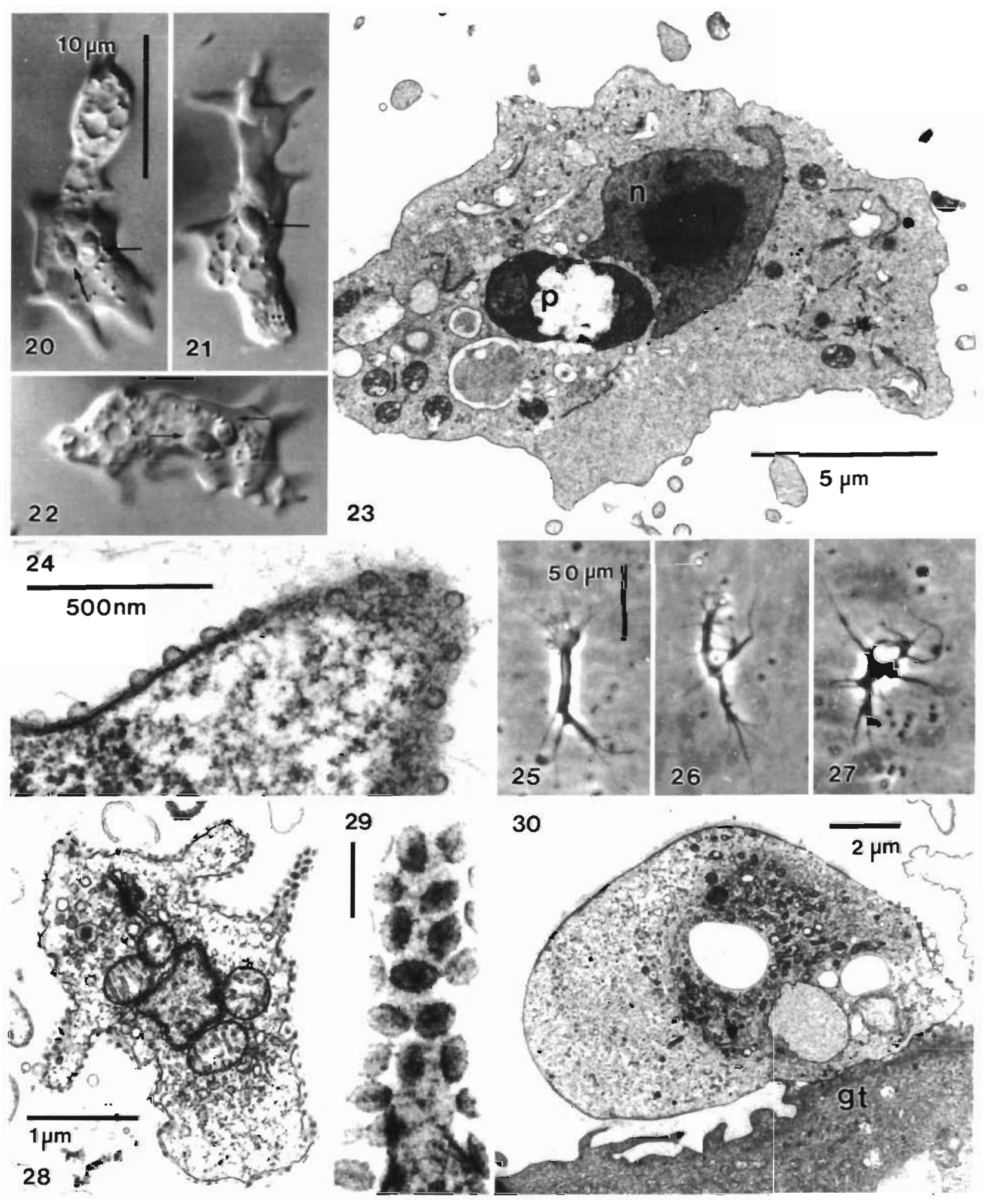

Figs. 20 to 30. Amoebae isolated from gills of turbots. Figs. 20 to 22. Trophozoites, representatives of 3 strains of Paramoeba sp. isolated from gills of turbots as seen in hanging-drop preparations. Arrows indicate parasomes. Fig. 23. Ultrathin section of a Paramoeba trophozoite with a parasome $(\mathrm{p}$ ) located in the close vicinity of the nucleus (n). Fig. 24. Trophozoite from primary isolates embedded for TEM with the cell surface structures resembling Pseudoparamoeba pagei. Figs. 25 to 27 . Branched buds of Corallomyxa observed in liquid medium used for primary isolations of amoebae from gills of turbot. Scale bar given in Fig. 25 Figs. 28 \& 29. Scale-bearing amoeba resembling Corythionella sp., and detail of scales. Scale bar = $200 \mathrm{~nm}$ in Fig. 29 Fig. 30. Mode of attachment of an unidenified amoeba trophozoite to the surface of gill tissue of turbot (gt) 
those of $P$. aestuarina Page, 1970, which according to original description by Page (1970) differs from $P$. pemaquidensis mainly in size.

In addition to the amoebae isolated and successfully cultured, 2 different species of amoebae were detected in material embedded for TEM from primary isolates growing on agar plates. The surface structures of one species (Fig. 24) resembled Pseudoparamoeba pagei Sawyer, 1975 as documented by Page (1983), while the second species was scale-bearing (Figs. 28 \& 29) and resembled Corythionella sp. (Euglyphidae), described by Golemansky (1974). Branched 'buds' of Corallomyxa were observed in liquid medium used for primary isolations of gill amoebae (Figs. 25 to 27). The amoebae populations from which our strains were derived can be taken for epizoites of the gill surface, as demonstrated by the firm attachment of one of the amoebae (unidentified in this case) to the gill surface (Fig. 30).

\section{DISCUSSION}

Although the results of our previous study of AGD in turbots (Dyková et al. 1995, 1998a) gave clear evidence that Paramoeba sp. was the agent of gill lesions, the simultaneous isolation of this and other species of amoebae from gills of clinically diseased turbots and gills of turbots of suboptimal health status, as well as other published data (Leiro et al. 1998), raises the question of the role of free-living amoebae other than Paramoeba sp. Predominance of flattened amoebae of discoid or fan shape among the strains isolated both from altered and clinically healthy gills of turbots also directs attention to the study of the potential pathogenicity of Platyamoeba, Vannella and Flabellula species.

The isolation of Paramoeba strains from clinically healthy gills and the fact that they only slightly differed from $P$. pemaquidensis and $P$. aestuarina provide compelling evidence that the taxonomy of free-living amoebae capable of invading the gills of turbots requires further careful studies.

The number of strains isolated from the gills of turbots with slight behavioral alterations and the number of recognized amoeba species indicated that species of some genera of flattened amoebae can easily colonize gills. In view of the lack of data on abundance of gymnamoebae in the water habitat, it is difficult to compare their density with the number of isolates from gill tissues. Curiously enough, some species declared to be most common among marine amoebae, e.g. Vexillifera telmathalassa or species of the genera Hartmannella, Neoparamoeba and Stygamoeba (Anderson 1994, Butler \& Rogerson 1995, Bradley \& Marciano-
Cabral 1996) were not identified among the isolated strains. Acanthamoeba species, too, were not reported from water samples taken in the area which partly supply the fish farms under study (Lloves et al. 1996). Since alternating patterns of abundance were observed between the groups of gymnamoebae including Acanthamoeba, Vexillifera and Mayorella on the one hand and Vannella and Platyamoeba species on the other (Anderson 1998), it would be of interest to have more data on marine habitats which supply water to turbot farms.

The negative results of isolation attempts achieved in some fish from each group as well as in all specimens of one group of fish kept in tanks supplied with water from a single source dismiss the possibility that free-living amoebae from residual water present on gills rather than amoebae from gill tissues were isolated. Moreover, direct proof of firm attachment of trophozoites on the surface of gills was given (Fig. 30).

Since the group of 20 fishes sent to the laboratory was declared to have an optimal health status and since a persisting effect of therapeutical baths could not be excluded, we do not overestimate the negative results of isolation attempts made in this group of turbots. We are convinced that more importance has to be placed on the presence of amoebae on gills of fish in suboptimal health condition.

While the pathogenicity of Paramoeba sp. (pemaquidensis?) was clearly demonstrated by the sequence of lesions caused in turbot gills, the evaluation of potential pathogenicity of other species of amoebae isolated from gills is more difficult. We have to take into account the nutritional requirements of amoebae, the adhesivness of trophozoites, which has been studied rather rarely (Custodio et al. 1995), and factors debilitating the health condition of fish. Valuable data can be accumulated when culturing amoebae on agar plates or in liquid media, while some factors affecting the host can hardly be assessed in natural infections. We can suppose that even epizoic amoebae, which prefer to feed on bacteria rather than on tissue debris, could harm the host when they multiply to huge quantities and use the gills as a support.

While we can prepare appropriate infective doses to design experiments, we are missing standard methods to induce immunosuppression of the host. Unless they are elaborated, we can hardly reproduce the gill disease under laboratory conditions. We are speculating on amoebae which otherwise are freeliving organisms and we need to study factors that compel free-living amoebae to become pathogenic parasites.

There is sound reason for assigning amoebae with flattened, mostly discoid, trophozoites to the genus level only. Light microscopy and TEM of glycocalyx 
allow the genus of the flattened amoebae to be determined rather safely. However, descriptions of named species of the genera Platyamoeba, Vannella and Flabellula show a wide variation in the ranges of length and breadth of trophozoites. This is a common feature in amoebae which makes species determination more difficult than in other protistan groups. The species of the above-mentioned genera do not form cysts or at least cysts are not known. For determination, the knowledge of type localities plays an important role in the case of extreme environments, while in free-living organisms it is probably of limited importance. For the time being, we prefer to circumvent the ultimate species determination of flattened, mostly discoid or flabellated amoebae by presenting examples of different well-established strains or clones. Since most of the strains isolated from turbots have been successfully cloned, it may be possible to characterize them in detail later (using both morphological and nonmorphological approaches) rather than increase the number of vague descriptions of allegedly new species now.

Species of Paramoeba appear comparatively to be the least difficult to classify. Only a few species were assigned to the genus and several strains of different origin were compared and their variation assessed by Page when he described $P$. pemaquidensis and $P$. aestuarina (Page 1970). In addition, we have our own promising experience from comparing trophozoites of different Paramoeba strains. The identity of $P$. eilhardi Schaudinn, 1896 is beyond suspicion because of the scale-bearing type of its glycocalyx; this helps to restrict the scope of Paramoeba species to be taken into consideration. Their final identification will be published elsewhere.

Acknowledgements. This research was funded by the Grant Agency of the Academy of Sciences of the Czech Republic (Project No. A6022802 and No. K2-022-601). The skillful technical assistance of $\mathrm{Mr}$ Jaroslav Bica was of great help.

Editorial responsibility: Wolfgang Körting,

Hannover, Germany

\section{LITERATURE CITED}

Anderson OR (1994) Fine structure of the marine amoeba Vexillifera thelamathalassa collected from a coastal site near Barbados with a description of salinity tolerance, feeding behaviour and prey. J Eukaryot Microbiol 41: $124-128$

Anderson OR (1998) Densities and diversity of gymnamoebae in relation to some inshore aquatic habitats at Bermuda J Eukaryot Microbiol 45:151-155

Bradley SG, Marciano-Cabral F (1996) Diversity of free-living 'naked' amoeboid organisms. J Indust Microbiol 17: $314-321$

Butler H, Rogerson A (1995) Temporal and spatial abundance of naked amoebae (Gymnamoebae) in marine benthic sediments of the Clyde Sea Area, Scotland. J Eukaryot Microbiol 42:724-730

Custodio MR, Imsiecke G, Borojevic R, Rinkevich B, Rogerson A, Müller WEG (1995) Evolution of cell adhesion systems: evidence for Arg-Gly-Asp-mediated adhesion in the protozoan Neoparamoeba aestuarina. J Eukaryot Microbiol $42: 721-724$

Dyková I, Figueras A, Novoa B (1995) Amoebic gill infection of turbot, Scophthalmus maximus. Folia Parasitol 42:91-96

Dyková I, Figueras A, Novoa B, Fernandez-Casal J (1998a) Paramoeba sp., an agent of amoebic gill disease of turbot. Scophthalmus maximus. Dis Aquat Org 33:137-141

Dyková I, Lom J, Macháčková B, Pecková H (1998b) Vexillifera expectata sp. n. and other non-encysting amoebae isolated from organs of freshwater fish. Folia Parasitol 45: $17-26$

Golemansky $V$ (1974) Psammonobiotidae fam. nov.-une nouvelle familie de thecamoebiens (Rhizopoda: Testacea) du psammal supralittoral des mers. Acta Protozool 13 $137-141$

Leiro J, Paniagua E, Ortega M, Parama A, Fernandez J, Sanmartin $M$ (1998) An amoeba asssociated with gill disease in turbot, Scophthalmus maximus (L.). J Fish Dis 21 $281-288$

Lloves M, Lores B, Pascual S, Arias C, Paniagua E (1996) Isolation of Acanthamoeba spp. in intensive aquaculture areas of Vigo estuary (NW, Spain). Sci Mar 60:549-551

Page F (1970) Two new species of Paramoeba from Maine. J Protozool 17:121-127

Page F (1980) Fine structure of some marine strains of Platyamoeba (Gymnamoebia, Thecamoebidae). Protistologica 16:605-612

Page F (1983) Marine Gymnamoebae. Institute of Terrestrial Ecology, Cambridge, p 23-45

Submitted: March 24, 1999; Accepted: June 28, 1999

Proofs received from author(s): September 20, 1999 